\title{
RIVOLUZIONE ALIMENTARE E PARCHI AGRICOLI \\ MULTIFUNZIONALI NELLA PIANA FIORENTINA
}

\author{
FOOD REVOLUTION AND THE MULTIFUNCTIONAL \\ AGRICULTURAL PARK IN FLORENTINE PLAIN
}

\author{
di Daniela Poli
}

\section{Jel Classification: R11, Q18, R58}

Le città occidentali si stanno lentamente riorganizzando in conseguenza dell'uscita dall'industrializzazione e della crisi del modello agroindustriale. La aree periurbane sono investite da una dinamica di riqualificazione che vede l'utilizzo di strumenti come i parchi agricoli multifunzionali, che hanno acquistato recentemente un connotato etico rilevante sulla spinta di vari "movimenti del cibo". Nell'articolo verranno illustrati i capisaldi della "grande rivoluzione del cibo"; la centralità del periurbano come contesto in cui le tematiche della sostenibilità, della sicurezza alimentare e della giustizia sociale a lungo disgiunte sono riunite grazie alla territorializzazione delle politiche del cibo e infine il progetto Coltivare con l'Arno. Parco agricolo perifluviale nella Piana fiorentina in cui questi aspetti sono stati trattati in ottica integrata, multifunzionale e contrattuale.

Parole chiave: pianificazione del cibo, periurbano, governance, sovranità alimentare, multifunzionalità, parco agricolo

Western cities are gradually reorganising as a consequence of the end of industrialisation and the crisis of agro-industrial model. Peri-urban areas are involved in redevelopment dynamics using innovative tools as multifunctional agricultural parks, which recently assumed a major ethical nuance in the wake of movements for food. The paper illustrates: the cornerstones of such a "great food revolution"; the centrality of peri-urban areas as the context where the issues of sustainability, food security and social justice, separated since long, are reconnected through the territorial dimension given to food policies; finally, the project Farming with Arno. Riverside Agricultural Park in the Florence Plain, in which these aspects have been approached in an integrated and multifunctional perspective based on negotiation.

Key words: Food Planning, Food Sovereignty, Peri-urban Area, Governance, Multifunctionality, Agricultural Park 


\section{Premessa}

La riorganizzazione dei territori periurbani ha subito una rilevante qualificazione grazie ai temi della sovranità e della giustizia alimentare che hanno assunto negli ultimi anni un ruolo centrale sia nel dibattito scientifico sia nelle vertenze sociali dei territori locali. Come noto, i dati generali sulla fame del mondo denunciano l'accesso ineguale al cibo su scala planetaria e una malnutrizione responsabile di quasi la metà dei decessi di bambini al di sotto dei cinque anni. La Fao ha dichiarato che nel 2009 per la prima volta nella storia il numero delle persone che soffrono la fame ha sorpassato la soglia simbolica del miliardo ${ }^{1}$. Ai più di $1.000 \mathrm{~kg}$ annui di cereali (compresa l'alimentazione animale per fornire carne) a disposizione di un abitante del Nord America non corrispondono neanche $200 \mathrm{~kg}$ per un africano (Grillotti Di Giacomo, 2012). Anche la corsa alla sostenibilità tramite la coltivazione di biocarburanti aggrava la disparità fra Nord e Sud del mondo. Non solo si distruggono contesti paesaggistici di pregio, ricchi di biodiversità che rappresentano il tessuto capillare dell'economia di sussistenza per le popolazioni locali, ma si arriva al paradosso che per fare il pieno a un veicolo tipo Suv di medie dimensioni occorre una quantità di cereali pari a quella che può coprire l' alimentazione annuale di una persona. Così mentre al Nord c'è chi fa il pieno per l'auto e al Sud c'è chi non riesce ad alimentarsi e vede distruggere la materia prima necessaria al proprio sostentamento e con essa i saperi contestuali incorporati nel territorio per lasciare spazio alle coltivazioni energetiche.

Anche su scala locale il problema dell'ingiustizia alimentare assume toni diversi, ma è drammaticamente presente. Da un'indagine Eurostat del 2011 emerge come in Europa la povertà stia aumentando. Nel 2010 circa il $23 \%$ della popolazione dei 27 paesi UE era a rischio di povertà o di esclusione sociale, e fra questi il $27 \%$ dei minori risultava colpito da povertà (Caritas Italiana, 2012). I dati mostrano come sempre più italiani (pensionati, disoccupati, famiglie con bambini piccoli) ricorrano agli aiuti alimentari per indigenti e si rivolgano alle mense della Caritas. Nel 2014, ad esempio a Taranto, gli utenti delle mense gestite dalla Caritas sono significativamente aumentati rispetto agli anni precedenti.

Visto che viene prodotto più cibo di quanto sia necessario per alimentare la popolazione mondiale, che pur risulta raddoppiata fra 1960 e 2003, è ormai palese che la fame non è conseguenza della scarsità di cibo. La produzione agricola mondiale è aumentata di $2,5-3$ volte negli ultimi 50 anni.

1 <http://www.fao.org/news/story/fr/item/20690/icode>. 
Dal 1961 al 2009 si passa da 2.189 kcal pro capite a 2.831 con un incremento del 29\%. «La disponibilità teorica media eccede quindi il fabbisogno medio di assunzione di calorie» (Sonnino, 2014: 8).

Il problema è spiegabile, piuttosto, con l'ineguale distribuzione causata dalla modalità di produzione industrializzata del cibo legata alle catene lunghe di produzione-distribuzione-consumo. La globalizzazione alimentare tiene infatti il mondo della produzione separato da quello del consumo tramite poche grandi società di mediazione che si occupano di commercializzazione, orientano le politiche internazionali e definiscono i prezzi. I grandi $h u b$ del cibo traggono il maggior beneficio senza portare vantaggi generalizzati, costringendo i piccoli agricoltori a restare esclusi dal mercato a causa dei prezzi troppo bassi di acquisto.

Fig. 1a - Produzione globale mostra la separazione e la gestione dell'interscambio da parte di società globalizzate, i grandi hub del cibo

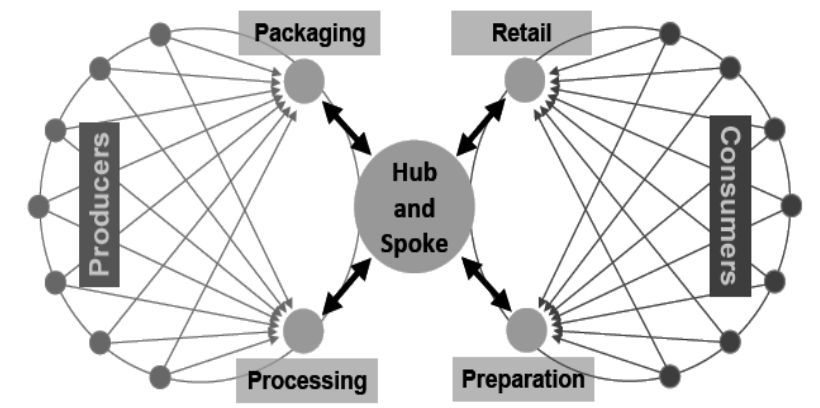

Fig. $1 b$ - Produzione locale che mostra un percorso interconnesso senza dei passaggi nessuna mediazione per la distribuzione

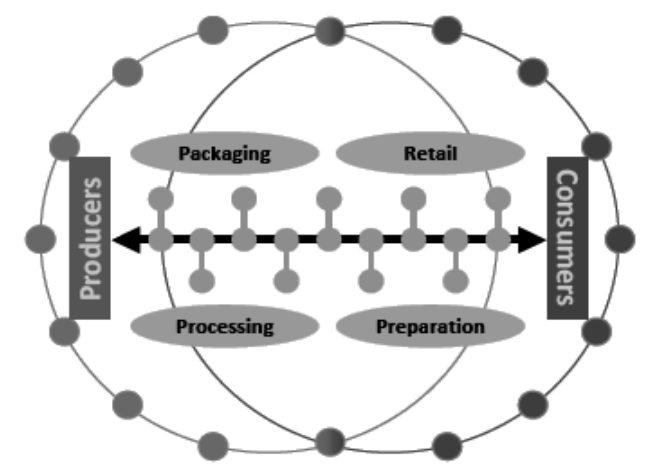

Fonte: Healthy Agroecosystems and Sustainable Communities 
Il paradosso è che il cibo delle reti lunghe non arriva in molti Paesi del Sud del mondo che non raggiungono neanche i requisiti minimi di sicurezza alimentare (food security) $)^{2}$, mentre quello abbondante che campeggia nei supermercati del Nord è insicuro (food safety) perché inappropriato dal punto di vista nutrizionale, qualitativo, culturale ed è banalizzato e ricco di grassi.

In molte città occidentali è iniziata dal basso un'inversione di tendenza, volta all'eliminazione dell'intermediazione nella catene alimentari per saldare il più possibile la produzione al consumo. Ciò che fino a pochi anni orsono era trattato dall'opinione pubblica in termini pietistici (la fame nel mondo) è diventato un elemento catalizzatore di domanda di politica alimentare più equa e sostenibile che trova un risvolto importante nella pianificazione urbana del cibo (Pothukuchi and Kaufman, 1999).

\section{Il periurbano catalizza i movimenti del cibo che si colorano di giustizia alimentare e di riqualificazione ambientale}

Nelle aree di vecchia e nuova urbanizzazione è in atto una ribellione sociale che intende capovolgere il sistema globalizzato del cibo, ripartendo da un nuovo modo di produrre, di commercializzare e di intendere l'alimentazione, riallacciando i rapporti fra città e campagna.

Il tema dell'agricoltura urbana ha fatto il suo ingresso nelle città occidentali sulla spinta dei "movimenti del cibo" i food movement, nati negli Stati Uniti in un contesto largamente insicuro perché dipendente dall'agroindustria con una forte incidenza di problemi sanitari legati allo scarso valore nutritivo e all'alta presenza di cibi addittivati (Feagan, 2007; Pollan, 2006; Paddeu, 2012). I movimenti del cibo hanno spinto alla definizione di politiche pubbliche come il Food Policy Concil, istituito per la prima volta nel 1982 a Knoxville per dare risposta al problema del deserto alimentare della città (Stierand, 2012: 71). Il "ceto medio riflessivo" (Ginsborg, 2010) inneggia al cibo biologico e locale e stigmatizza negativa-

2 La food security, come riconosciuto dalla Fao è un concetto evolutivo ed è presente in circa 200 diverse definizioni. Oltre alla definizione iniziale del 1974 del World Food Summit, vi sono stati aggiornamenti a metà degli anni ' 80 e nel 1996. Inizialmente, l'attenzione era posta sui problemi di fornitura di cibo, al fine di assicurare la presenza stabile e a prezzi equi di cibi di base a livello sovranazionale e nazionale <http://www.ttsecurity.net/ 2011/02/04/sicurezza-e-salute-del-cibo-nella-societa-del-rischio>, poi il concetto si è evoluto fino a specializzarsi anche in quello di food safety. Come noto, nel Nord America in molti casi si sfiora l'insicurezza alimentare perché molte aree sono prive di luoghi di commercializzazione del cibo e nei cosiddetti deserti alimentari non si trovano i supermercati. 
mente il comportamento degli industrial eaters, che consumano cibo trasformato dall'industria agroalimentare (Pollan, 2006). Non casualmente nel 2007 appare sull'Oxford English Dictionnary il termine locavore per designare la persona che si ciba di alimenti locali.

Complice la crisi economica si diffonde nelle città occidentali sempre con maggior vigore la domanda non solo di sicurezza, ma di autogoverno delle politiche alimentari, con la richiesta di incidere direttamente sull'organizzazione della filiera della produzione e consumo del proprio territorio facendo appello alla "sovranità alimentare"3. Negli Stati Uniti si ridisegnano i territori sulle Community food security (Cfs) intese come «un processo di rispazializzazione dei sistemi del cibo organizzato sulla delimitazione spaziale a partire dalla comunità sociale» (Feagan, 2007: 27). Emerge così sempre con maggiore forza il ruolo dell'agricoltura di piccole dimensioni che ritesse relazioni, produce cibo sano e lo commercializza tramite reti formali e informali che coinvolgono in maniera equa le comunità di cittadini.

Le città non solo si ruralizzano (Mougeot, 2005; Donadieu, 2006 e 2011), ma si caratterizzano per essere potenti agenti di sviluppo rurale che legano la città alla campagna (Oecd, 2013), assecondando i nuovi stili di vita e di consumo, talvolta anche insorgenti che mettono al centro della propria azione un sistema di valori solidale con la società e con l'ambiente (Potito e Borghesi, 2015; Poli, icp). Sotto questa spinta le aree periurbane sono diventate dei vivacissimi laboratori sociali in cui si sperimenta un reincorporamento spaziale della catena alimentare (Feagan, 2007) organizzato in più scale, quella di prossimità e quella bioregionale.

La città di prossimità è quella che si vive tutti i giorni, una città sempre più edibile, dove con spostamenti minimi, utilizzando mezzi pubblici o mobilità dolce si può andare a comprare direttamente in azienda, nei tanti mercati contadini della città, negli incontri con i Gas, oppure si può coltivare nell'orto, passeggiare attraverso campi coltivati o accompagnare i fi-

3 Il principio della sovranità alimentare, nato in occasione del Forum parallelo al vertice Fao sulla Sicurezza Alimentare del 1996 (Fao 1966), si basa su 4 pilastri: diritto al cibo; accesso, gestione e controllo delle risorse naturali; modello agricolo sostenibile e di filiera corta; commercio internazionale più giusto e più equo. «La sovranità alimentare è il diritto delle persone a un cibo culturalmente appropriato e sano, prodotto con metodi sostenibili che rispettano l'ambiente ed il diritto a definire i propri sistemi agricoli e alimentari. La sovranità alimentare pone al centro dei sistemi e delle politiche alimentari le aspirazioni e i bisogni di coloro che producono, distribuiscono e consumano cibi, anziché le richieste delle aziende e dei mercati» (cfr. Declaration Of Nyéléni, Nyéléni Village, Sélingué, Mali 27 February 2007 <www.nyeleni.org/IMG/pdf/DeclNyeleni-en.pdf>). 
gli a scuola e osservarli piantare frutti nell'orto scolastico. Il cibo è sempre più un'occasione di incontro e di socializzazione che domanda una riqualificazione degli spazi di vita.

La scala bioregionale è quella più ampia è il bacino del cibo sociale il food shed, descritto come «uno spazio socio-geografico con attività umane incorporate nella membrana naturale di un particolare luogo» (Kloppenburg, Hendrickson and Stevenson, 1996: 37), una metafora che aiuta a delineare i contorni di questa decisiva riprogettazione spaziale su base locale. La "rivoluzione copernicana del cibo" è infatti impossibile da attuare individualmente, ma si fonda su collettività autoriconosciute che condividono momenti di acquisto, di co-produzione, di scambio, di diffusione dei saperi. La connessione fra l'alimentazione e la forte richiesta di giustizia sociale è in grado di aprire nuove porte a un immaginario, che superi il dominio del tradizionale binomio sociale ed economico (Soja, 2009) per traghettarlo verso la giustizia alimentare (Gottlieb and Joshi, 2010). La rivoluzione copernicana del cibo porta così alla riconfigurazione del contesto periurbano elevandolo al rango di «spazio pubblico bioregionale» (Poli, 2014), dove vivere, produrre manutenendo l'ambiente e il paesaggio e consentendo ai fruitori di riacquistare il piacere della condivisione con l'altro (Parham, 2015).

\section{Ricostruire prossimità con un progetto integrato e multi-funzionale: il caso di "Coltivare con l'Arno. Parco Agricolo Perifluviale" nella pianura fiorentina di Riva sinistra d'Arno}

Centrare l'attenzione sulla dimensione "locale" delle reti agricole ha portato alla riconnessione tra le grandi tematiche della sostenibilità ambientale, della sicurezza alimentare e della giustizia sociale (Paddeu, 2012). Riterritorializzare i sistemi del cibo è per le società contemporanee un obiettivo prioritario da raggiungere con un progetto integrato che agisca contemporaneamente su più aspetti, quali:

- la resilienza insediativa per consentire l'adattamento ai cambiamenti climatici;

- la sostenibilità ambientale per garantire qualità della vita e delle produzioni;

- l'economia circolare che minimizza i rifiuti e ottimizza la catena valoriale della produzione;

- $\quad$ il rafforzamento della progettualità sociale verso l'autogoverno della comunità del cibo; 
- la coproduzione del cibo e la co-governance delle politiche urbanorurali tramite accordi fra Pubbliche amministrazioni, agricoltori e cittadini;

- la giustizia alimentare includendo le popolazioni più fragili e individuando il giusto prezzo agli alimenti di filiera corta;

- la qualità del paesaggio per garantirne la fruizione consapevole a cittadini e turisti.

Con questo spirito si stanno susseguendo progetti istituzionali e progetti autorganizzati per la realizzazione di parchi agricoli che animano un ricco dibatto sociale e che si rivelano utili per la definizione di una pianificazione urbano-rurale di nuova generazione sistemica, multilivello, integrata e intersettoriale in cui il ruolo plurimo dell'agricoltura possa dialogare attivamente con la complessità delle dinamiche territoriali, le comunità insediate i mercati locali (Bocchi, 2015) e non ultimi gli strumenti di pianificazione territoriale. Mentre in riva destra d'Arno recentemente (2014) è stato istituito a livello regionale il perimetro del parco agricolo della Piana e nel comune di Prato è attiva un'associazione per la promozione del parco agricolo di Prato che ha già raggiunto diversi obiettivi, fra cui la filiera cerealicola del pane, il progetto Coltivare con l'Arno. Parco agricolo perifluviale è un processo partecipativo che ha ottenuto il sostegno dell'Autorità Regionale per la Garanzia e la Partecipazione della Regione Toscana (lr 46/2013) nei territori periurbani di Riva sinistra d'Arno fra Firenze, Scandicci e Lastra a Signa.

Il progetto ha preso avvio con un protocollo d'intesa per la valorizzazione dell'agricoltura periurbana (2012) che coinvolgeva le Amministrazioni locali e il mondo dell'università ${ }^{4}$. L'approccio di tipo bioregionale (Iacoponi, 2001; Thayer, 2003; Calthorpe and Fulton, 2001, Magnaghi, 2014a e 2014b; Frenkel, 1994; McTaggart, 1993; Meredith, 2005) ha delineato un progetto integrato per riconfigurare l'area di prossimità (Mincke

4 Il protocollo d'intesa per la valorizzazione dell'agricoltura periurbana era stato sottoscritto dalla Regione Toscana, dalla città metropolitana, dai Comuni di Firenze, di Scandicci e di Lastra a Signa assieme alle Facoltà di Architettura e di Agraria (2012). La ricerca, Coltivare con l'Arno. Parco agricolo perifluviale, è coordinata dall'autrice e condotta da un gruppo di ricercatori, laureandi e tirocinanti <www.coltivareconlarno.it〉. Il gruppo di lavoro universitario è affiancato da un Comitato Scientifico Multidisciplinare dell'Università di Firenze coordinato da Alberto Magnaghi, che fa capo all'Unità di Ricerca Progetto Bioregione urbana <http://www.dida.unifi.it/vp-323-probiur.html> cui appartengono urbanisti, agronomi, forestali, naturalisti, economisti. 
et Hubert, 2011) nel territorio intermedio fra le aree urbanizzate, le colline e l'Arno, coinvolgendo ampi settori della società e delle parti sociali.

Il territorio che fino agli anni '50 era indicato come 1' "orto di Firenze" è caratterizzato oggi da forti contrasti: paesaggi collinari di pregio ampiamente gentrificati, pianura largamente urbanizzata dove vive la maggior parte della popolazione con la presenza di fasce di basso reddito e di immigrazione, aree agricole intercluse che affacciano sul fiume difficilmente raggiungibile, ma sul quale molti gruppi sociali stanno progettando attività. La forte percentuale di suolo urbanizzato nel territorio di pianura fra i comuni di Firenze, Scandicci e Lastra a Signa, che raggiunge nel 2007 il 60,5\% (Butelli, 2015), ha indotto le Amministrazioni a individuare negli strumenti di governo del territorio le aree a "parco agricolo" in prossimità del fiume per frenare la nuova urbanizzazione. La tutela dei territori periurbani è recentemente stata rafforzata dall'entrata in vigore dell'innovativa legge regionale 65/2014 in materia urbanistica che sancisce l'impossibilità di edificare edifici residenziali al di fuori del limite dell'urbanizzato, definendo con chiarezza la separazione fra territorio urbano e territorio rurale.

Il lavoro ha preso avvio dall'obiettivo generale di progettare un parco agricolo multifunzionale che si estendesse dal fiume alle colline come progetto pilota del "Contratto di Fiume" $(\mathrm{CdF})$ dell'Arno e del futuro piano strategico triennale della città metropolitana. Gli obiettivi principali erano incentrati sulla definizione del Sistema Agroalimentare Locale, sulla riqualificazione ambientale, sul rafforzamento delle reti corte, sulla definizione di uno scenario strategico e sull'individuazione di azioni prioritarie per la definizione del primo piano d'azione 5 .

Il progetto ha raffinato nel corso dei lavori gli obiettivi generali che hanno ottenuto un carattere viepiù operativo grazie a una metodologia di ricerca-progetto-azione, che ha costantemente interagito con i soggetti lo-

5 Definire un parco agricolo perifluviale come laboratorio sperimentale di innovazione politica e sociale; ridare un ruolo strategico ai fiumi; riconoscere il ruolo strategico dell'agricoltura come mediatrice di riappropriazione; progettare in maniera integrata per un'agricoltura multifunzionale produttrice di cibi sani e stili di vita salubri; definire il Sistema Agroalimentare Locale; nutrire la città metropolitana fiorentina; favorire l'accesso alla terra delle nuove generazioni; contribuire a regolare l'equilibrio idrogeologico dell'area; sostenere la transizione verso un'agricoltura sostenibile, sana e di qualità; attrezzare dal punto di vista logistico il parco agricolo; avviare azioni di sostegno pubblico alle attività e al commercio agroalimentare locale; favorire il ruolo dell'ente pubblico nell'affidamento di attività e servizi agli agricoltori; restituire ai centri urbani e alle città l'affaccio sul fiume, attraverso la mediazione dell'agricoltura; ripensare il sistema dell'accessibilità e della mobilità nel parco agricolo e sul fiume; coniugare agricoltura e lavoro sociale. 
cali per diffondere consapevolezza e conoscenza, ma soprattutto per costruire proposte operative condivise.

Il progetto ha inteso sperimentare l'integrazione fra la dimensione pattizia del "Contratto di Fiume" (Bastiani, 2011) ${ }^{6}$ e la progettualità integrata del Parco agricolo multifunzionale (Magnaghi e Fanfani, 2010), con l'obiettivo strategico di definire un "Contratto di Fiume" a valenza di Parco agricolo perifluviale (Poli, 2015).

Il sostegno ottenuto dall'Autorità per la Partecipazione ha consentito un confronto ampio e diffuso con le tante soggettività locali, includendo i soggetti deboli e le organizzazioni sociali innovative ma scarsamente riconosciute a livello istituzionale come il Comitato di autogestione delle terre pubbliche a Lastra a Signa che sta gestendo in forma condivisa un orto sociale di circa 3,5 ettari col progetto Guerrina Terra Sociale. ${ }^{7}$

Il processo ha visto un doppio livello di governance:

- I livello: Tavolo d'Area con rappresentanti istituzionali e delle associazioni;

- II livello: Laboratori locali con abitanti, agricoltori, associazioni locali.

Il lavoro del Tavolo d'Area è servito soprattutto per definire una struttura allargata di pilotaggio del progetto con la quale condividere le orientazioni strategiche. Una delle prime scelte del Tavolo d'Area ${ }^{8}$ è stata quella di estendere l'area del parco agricolo, passando dai circa 500 ettari di territorio agro-forestale, tutelati dagli strumenti di governo locale del territorio situati nel contesto perifluviale, ai circa 5.000 ettari comprensivi dei rilievi collinari retrostanti, caratterizzandola in tal modo come un sottobacino del fiume Arno. Il lavoro con i Laboratori locali è stato un "corpo a corpo" con le conoscenze contestuali emerse negli incontri di ascolto attivo e di progetto ed è servito per allargare lo spettro delle informazioni e delle oppor-

6 Il Ministero dell'Ambiente ha riconosciuto istituzionalmente i CdF nell'art. 24bis "Contratti di Fiume" del Codice dell'Ambiente (dlgs 152/2006). Rifacendosi alla Convenzione Europea del Paesaggio, la riqualificazione fluviale è intesa in senso ampio e prevede intersettorialità e interrelazione fra più aspetti (idrogeomorfologici, ecologici, insediativi, rurali, fruitivi, partecipativi, estetici, ecc.) ed è tesa all'elaborazione di scenari di sviluppo durevole in cui dal minuto tessuto insediativo locale.

7 Il progetto nasce dalla collaborazione tra il Comitato Terre di Lastra Bene Comune e l'Associazione Culturale Voltapagina, con il consenso dell'Amministrazione comunale e coinvolge molte realtà locali <https://www.facebook.com/terrelastrabenecomune/notes >

8 Fra gli altri: l'Autorità di Bacino, il Consorzio Risorse Idriche, l'Istituto Penitenziario di Sollicciano e di Solliccianino, l'Istituto Tecnico Agrario, il Cai, l'Uisp, le Pro Loco, il Comitato Terra Bene Comune, Legambiente, Il Centro Italiano di Riqualificazione Fluviale, Slow Food, il Siaf, il Comitato dell'Isolotto, Italia Nostra. 
tunità. Il costante lavoro di rielaborazione e di nuova condivisione fra Tavolo d'Area e Laboratori locali ha messo in luce una nuova e ricca narrazione del territorio scritta a più voci dalla quale sono emerse innumerevoli potenzialità e anche molte criticità fino ad oggi inesplorate e soprattutto inascoltate dai vari Enti di riferimento.

Fig. 2 - La struttura del processo partecipativo

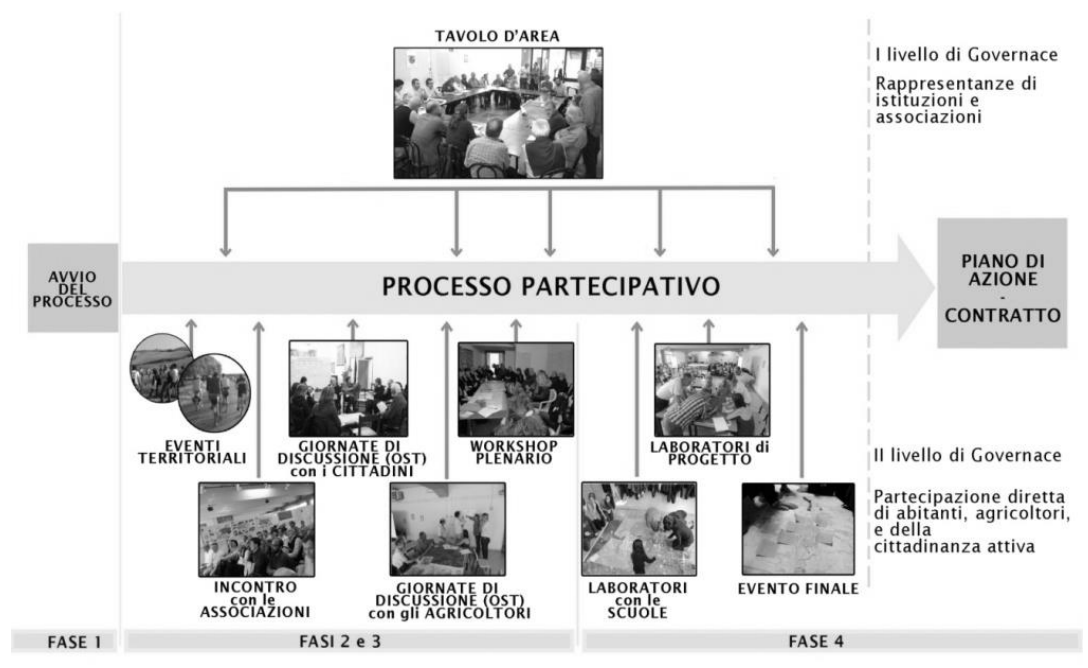

Fonte: Coltivare con l'Arno. Parco agricolo perifluviale, Progetto partecipativo in corso, coordinato da D. Poli con elab. grafica di E. Butelli

Il progetto si è avvalso della metodologia della ricerca-progetto-azione in un percorso fatto da un fluire continuo fra conoscenze tecniche e conoscenze contestuali, ipotesi, verifiche progettuali e condivisioni. Dopo una prima fase di ascolto attivo si è passati alla definizione contestuale di prefigurazioni spaziali e "contratti sociali", accordi di tipo volontario stipulati fra Enti pubblici (Amministrazioni comunali, Amministrazioni territoriali, Enti di gestione, come l'Autorità di Bacino, il Consorzio di bonifica, le Scuole, gli Ospedali, gli Istituti di Pena, ecc.), soggetti privati, e privato sociale (associazioni, gruppi sociali ecc.). L'idea di fondo è quella della co-progettazione e della co-gestione in modo che tutti i soggetti, pubblici, privati e associativi si sentano coinvolti e messi nella condizione di disponibilità all'ascolto reciproco per l'individuazione di soluzioni condivise che i sottoscrittori istituzionali del progetto dovranno impegnarsi nel rendere operative ciascuno nel proprio settore. Anche nelle politiche del cibo l'interdipendenza fra pubblico, privato e terzo settore è diffusamente rico- 
nosciuta come una metodologia molto più efficace e risolutiva che non il sistema verticale di governo del territorio (Barmier and Morin, 2012).

Il progetto è in corso di definizione e si propone di attivare linee di azione nel quadro del "Contratto di Fiume" da far sottoscrivere alle Amministrazioni locali con la previsione delle forme di gestione e dei finanziamenti attivabili. Fondamentale durante il percorso è apparso:

- far dialogare i soggetti attenti all'agricoltura sana e di qualità (agricoltori amatoriali, comitati genitori, Gas, ecc.) con gli agricoltori professionali;

- aumentare al consapevolezza locale sulle problematiche e sui valori del contesto di vita;

- rafforzare la cultura alimentare e sostenere la definizione di una comunità locale del cibo che si autoriconosca;

- utilizzare cartografie e scenari progettuali per la definizione condivisa delle strategie;

- dare un ruolo di leadership a soggetti o gruppi già attivi sul territorio;

- aumentare il livello di consapevolezza nelle Pubbliche amministrazioni.

Il progetto si compone di due parti che potrebbero trovare nello strumento orizzontale del Progetto Integrato Territoriale previsto dal Piano di sviluppo regionale 2014-20 della regione Toscana una fonte rilevante di supporto:

- uno scenario strategico di riqualificazione fisica del territorio;

- 12 contatti sociali fra soggetti pubblici, privati e associativi.

\subsection{Scenario strategico di riqualificazione fisica del territorio}

Lo scenario di riqualificazione fisica del territorio di prossimità consiste in una serie di azioni integrate che vedono come prerequisito fondamentale la riapertura delle relazioni ecosistemiche e la bonifica dei suoli, compromessi dalla presenza di aree industriali ora dismesse e da agricolture convenzionali intensive sul versante dei trattamenti chimici. L'approccio integrato intende rispondere a obiettivi plurimi per arrivare alla definizione di spazio pubblico alla scala bioregionale dove all'agricoltura multifunzionale si integrano più aspetti come la manutenzione del paesaggio, la riqualificazione dei fronti urbani, la definizione di luoghi di incontro urbano-rurali ognuno dei quali al tempo stesso svolge funzioni plurime e risponde a una logica multiobiettvo. Ad esempio il percorso di una nuova strada può svolgere la funzione di allontanamento del trasporto pesante dal centro storico e al tempo stesso quella di un'opera di mitigazione del rischio idraulico. 
La pianura mostra con evidenza i risvolti negative che l'industrializzazione dei mondi di vita e l'agroindustria hanno prodotto in poco più di cinquant'anni con una conurbazione che separa collina e pianura, con frammentazioni ambientali causate da una serie di infrastrutture parallele che la attraversano e suoli inquinati. La commercializzazione alimentare è monopolizzata dalla Gdo che ha prodotto un "deserto sociale" nelle relazioni fra cittadini e agricoltori che ora si sta rifertilizzando grazie all'attività dei Gas e a produzioni comunitarie del cibo.

Le stesse attività agricole appaiono settorializzate fra la campagna e la pianura. Nella pianura, ricca di elementi patrimoniali di pregio (centuriazione ancora evidente, canali, borghi rivieraschi di origine medievale, beni culturali come la Badia a Settimo), sono concentrate le attività orientate alla produzione orticola agroindustriale con aziende di medie dimensioni, con serre distribuite fra svincoli e viabilità di attraversamento. Sempre in pianura sono presenti molte piccole attività aziendali o amatoriali dedite all'orticoltura condotte prevalentemente da anziani che manifestano sofferenza nell'accesso ai contributi e nella prosecuzione delle attività. In collina si estende invece un paesaggio storico di pregio dove trovano posto molte aziende soprattutto olivicole e viticole con la presenza di servizi (agriturismi, vendita diretta, maneggi, ecc.) e molte aziende già dedite alla coltivazione biologica. Sebbene in tutto il territorio vi siano normative molto restrittive per quanto riguarda la nuova urbanizzazione sono presenti circa 200 ettari di terreno frammentato in molte parcelle e non coltivato, ma semplicemente mantenuto con minimi sfalci periodici in attesa di possibilità edificatorie; fra questi ricadono circa 35 ettari di terreno che rappresentano nell'insieme un'occasione rilevante per fornire l'accesso alla terra per le giovani generazioni di agricoltori o costruire opportunità per l'orticoltura amatoriale comunitaria.

Lo scenario strategico individuato per far rinascere dal punto di vista ecologico il territorio mira a ricollegare l'Arno e le colline con l'attraversamento della conurbazione tramite cunei verdi agro-ambientali che, come linfa vitale, penetrano nei luoghi, ricostruiscono margini e collegano i diversi ecosistemi. La parte centrale del parco si caratterizza per la presenza di tre ampi cunei verdi che dalla collina raggiungono il fiume Greve e l'Arno, postulando un "ridimensionamento" delle previsioni urbanistiche dei comuni che ad oggi sono sovradimensionati e spesso vanno proprio a occludere la aree ancora libere.

Il progetto restituisce un valore centrale all'Arno e ai suoi affluenti, che dovranno riconquistare il loro ruolo centrale di corridoi ecologici multifunzionali, collegati al tessuto insediativo storico e alla mobilità dolce. 
Fig. 3 - Lo scenario strategico del parco agricolo multifunzionale

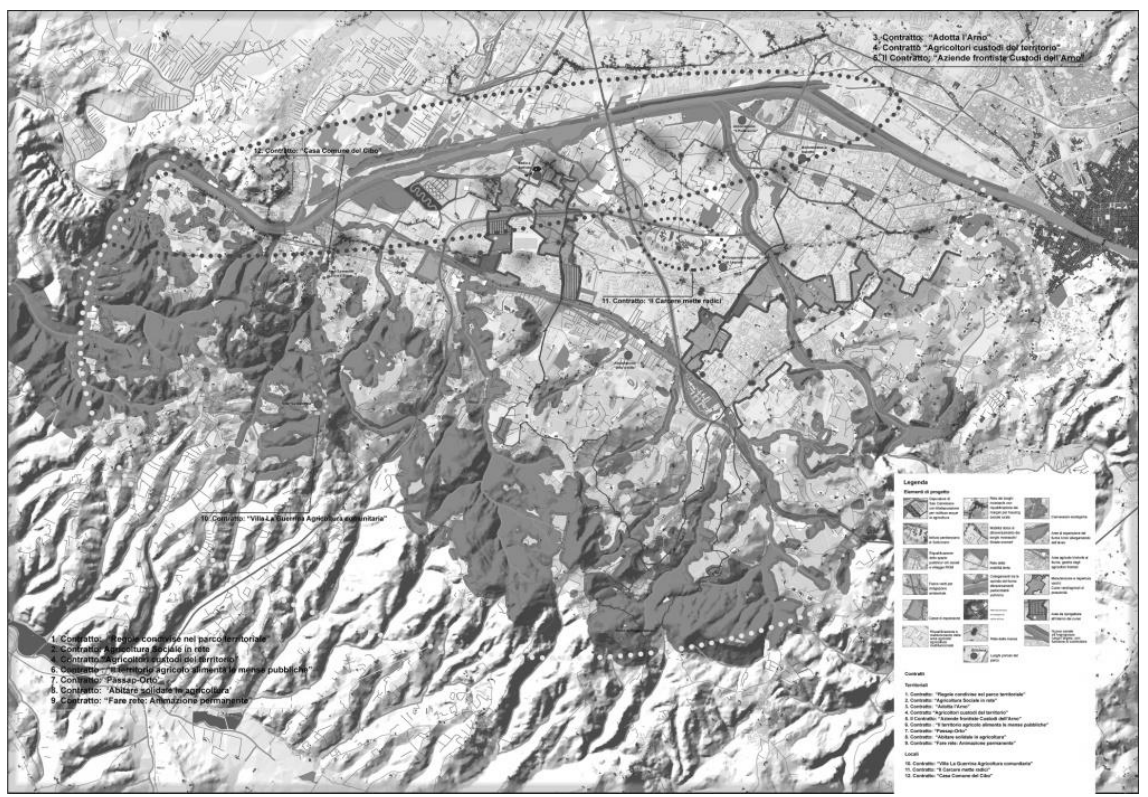

Fonte: Coltivare con l'Arno. Parco agricolo perifluviale, Progetto partecipativo in corso, coordinato da D. Poli con elab. grafica di E. Butelli

L'Arno, in particolare, deve tornare a svolgere il ruolo di ossatura portante del punto di vista ecologico regionale, recuperando per quanto possibile il proprio spazio di dinamica naturale, secondo quell'approccio che vede sviluppare in tutto il mondo una progettazione volta alla riqualificazione fluviale per restituire a fiumi e torrenti arginati gli spazi in cui l'acqua possa divagare con un maggior grado di libertà: dall'allargamento dell'alveo, alla definizione di aree golenali e zone di laminazione. I corsi d'acqua saranno affiancati da percorsi pedonali e ciclabili che non inficino la funzionalità ecologica del fiume, così come da aree di sosta e di ristoro con "spiaggette" ghiaiose formate dai processi erosivi del fiume. Fra i percorsi di mobilità dolce acquistano particolare rilevanza quelli legati alla navigabilità leggera del fiume Arno sia di carattere turistico che di linea, con la finalità principale di alleggerire il traffico su gomma e di riaprire le relazioni fra cittadinanza e fiume. Nell'ipotesi si prevedono diverse fermate del battello in corrispondenza degli attraversamenti sull'Arno che potranno così diventare nodi di socialità, con spiaggette e luoghi di sosta, gestiti dagli agricoltori frontisti. Fra i progetti di economia circolare legati all'acqua è da menzionare quello sul riuso a fini agricoli delle acque del 
depuratore di San Colombano che, ad oggi, vengono invece restituite direttamente al fiume.

Un territorio risanato tramite una nuova agricoltura invita cittadini, turisti- e visitatori a penetrare nella campagna per fare compere, svagarsi, correre fra i campi (Poli, 2013). Il parco sarà così innervato di percorsi di mobilità dolce che potranno penetrare anche all'interno delle viabilità poderali grazie alla valorizzazione della sentieristica storica. Lo scenario prevede attraversamenti pedonali e ciclabili dalla pianura alla collina, così come la realizzazione di woonerf 9 che attraversano i centri storici rivieraschi. I woonerf costituiscono localmente una percorrenza privilegiata di collegamento in sicurezza dei piccoli borghi sull'Arno dai quali si potrà raggiungere l'Arno per attraversare l'altra sponda.

Nel cuore interno dello spazio pubblico bioregionale sono stati definiti una serie di progetti finalizzati a rendere il territorio periurbano un contesto adatto ad ospitare un'agricoltura sana, di filiera corta, in grado di rispondere alle tante domande urbane che provengono da più settori e che fanno fatica a trovare risposte sul territorio.

\subsection{Un progetto locale e contrattuale}

Il progetto integrato di costruzione di un territorio intermedio di prossimità dovrà attuarsi tramite diversi "contratti" in via di definizione costruiti tramite una densa attività di co-governance verticale e orizzontale che ha visto incontri serrati con più soggetti quali Regione, Soprintendenza, Amministrazioni comunali, Società della Salute, Consorzio di Bonifica, Autorità di Bacino, Auser, Enti penitenziari, Apab, Associazioni di Categoria, ecc. I vari incontri hanno tessuto sempre nuove reti fra i partecipanti alimentando fiducia e rafforzando la progettualità locale. Il processo ha delineato per adesso 12 contratti, alcuni dei quali andranno a comporre il primo piano d'azione del Contratto di Fiume. Uno dei contratti è generale, di carattere normativo, mentre gli altri possono essere raggruppati in alcune azioni principali. Si raggiungono quindi: semplificazione normativa, sostegno alla multifunzionalità, opportunità dell'agricoltura sociale

\subsubsection{Semplificazione normativa}

1) Il contratto Regole condivise nel parco territoriale prevede una governance verticale fra istituzioni locali e associazioni di categoria agricole per

9 I woonerf, concepiti nei Paesi Bassi e diffusi in tutta Europa, si caratterizzano per essere strade nelle quali hanno precedenza i pedoni, dove si può sostare e giocare grazie alla riconfigurazione della carreggiata a questo fine concepita. 
condurre le procedure edilizie da una logica autorizzativa a una regolativa che preveda il ricorso a regole chiare e condivise fra i vari attori per consentire le diverse operazioni che gli agricoltori debbono sostenere (tettoie, serre, pollai, annessi, ampliamenti, ecc.) tramite l'uso di manuali e normative figurate che facciano riferimento alle morfotipologie insediative con tempi certi d'attuazione al posto di processi autorizzativi lunghi, incerti e discrezionali.

\subsubsection{Sostegno alla multifunzionalità}

Ci sono un gruppo di contratti finalizzati a sostenere e rafforzare le attività degli agricoltori tramite la costruzione di fonti di finanziamento integrative che valorizzino la multifunzionalità.

2) Il progetto Agricoltori Custodi del Territorio è finalizzato sostenere la funzione ambientale e di presidio dell'agricoltura locale, fornendo fonti di reddito integrative mediante il contributo per la gestione della rete idrica minore e della rete sentieristica, il supporto al turismo rurale e alla didattica e sostenendo, inoltre, l'inserimento lavorativo dei soggetti svantaggiati, la partecipazione alle reti di commercializzazione locale nei Gas o nella rete delle mense pubbliche.

3) Il contratto Aziende frontiste Custodi dell'Arno aggiunge alle funzioni di custodia del territorio aziende agricole relative ai corsi d'acqua principali, in particolare dell'Arno, per rendere fruibile e sicuro il fiume tramite la manutenzione della vegetazione ripariale, il monitoraggio del fiume, la gestione degli approdi di un battello che dal porto di Signa potrebbe arrivare fino a Firenze, la vigilanza sugli attraversamenti (passerelle, chiatte, ecc.) e sulle spiaggette lungo il fiume, la gestione di un servizio di noleggio per biciclette. I contratti di custodia prevedono accordi con diversi Enti e Associazioni di Categoria. Un'opportunità fondamentale per il sostegno all'agricoltura periurbana viene dalla possibilità di contare su una domanda aggregata certa come quella che proviene dalle mense pubbliche delle scuole.

4) Il contratto Adotta l'Arno nasce dalla progettualità sociale di gruppi e associazioni locali integrandosi con l'attività delle aziende frontiste del fiume ed è finalizzato alla manutenzione/animazione di un tratto fluviale dell'Arno caratterizzato da condizioni di perifericità che aumentano laddove sono localizzate diverse aree degradate e alcune funzioni "scomode" allontanate dal centro città come il campo Rom. Il progetto è composto da una serie di "progetti low cost" (eventi, fruizione, piccole manutenzioni, ecc.) realizzabili col coinvolgimento attivo della popolazione locale e del mondo dell'associazionismo. 
5) Il contratto Il territorio agricolo alimenta le mense pubbliche intende promuovere l'utilizzo delle produzioni di olio e verdura locale nelle mense pubbliche con la costituzione di una rete incrementale di agricoltori che possa rifornirle. Per raggiungere questo obiettivo è necessario sensibilizzare le famiglie e gli amministratori sul problema dalle sicurezza e sul potere nutrizionale degli alimenti che provengono dal territorio locale; redigere un capitolato partecipato con tutti i soggetti interessati; individuare una struttura che gestisca la rete dei produttori per alimentare la ristorazione collettiva. Oltre ai Comuni sono necessari i Comitati genitori delle scuole e le aziende che gestiscono l'appalto delle mense pubbliche.

6) Il contratto Fare rete: Animazione permanente è finalizzato a dar continuità al progetto. Per un parco agricolo di nuova generazione, leggero dal punto di vista amministrativo, che non prevede un Ente Parco ma un Comitato di gestione partecipato, è fondamentale la presenza di animazione territoriale costante, finalizzata al consolidamento della rete $\mathrm{e}$ all'attivazione di progettualità sociale continua mettendo a sistema i vari attori, dai Gas alle mense, ecc.

7) Nel contesto periurbano rinnovato sarà fondamentale individuare una nuova logistica di supporto alle diverse attività. Un elemento centrale di tale logistica è stato individuato nella "Casa Comune del Cibo" che risponde alla necessità di tanti piccoli agricoltori di conferire a qualcuno i loro prodotti perché essendo spesso formati da un solo operatore non riescono contemporaneamente a lavorare la terra e vendere i prodotti tramite la vendita diretta. La Casa comune del cibo prevede la riconversione di un'area dismessa nel comune di Lastra a Signa (ex macelli) in una struttura con più funzioni legate alla "multifunzionalità" dell'agricoltura del parco. Oltre alle funzioni commerciali legate alla vendita e alla ristorazione con $\mathrm{i}$ prodotti agricoli del parco, dovrebbe caratterizzarsi come cuore urbanorurale di una nuova socialità che si lega intimamente al concetto di consumo critico e a forme di scambio e baratto di elementi materiali o del tempo (la banca del tempo contadino) o di competenze e di saperi contadini tra cittadini e agricoltori. Il parco ha già attivato una sorta di scuola contadi$n a$, un corso di autoformazione sull'orticoltura biologica nel parco gestito dagli agricoltori e dalle associazioni locali.

\subsubsection{Le opportunità dell 'agricoltura sociale}

Un tema centrale e rilevante che chiama direttamente in causa la dimensione della giustizia sociale è quello delle molte e ampie opportunità di reinserimento e di riscatto che l'agricoltura sociale offre, grazie anche alla 
recente legge nazionale in materia ( $\mathrm{dl}$ 141/2015). Il progetto prevede tre contratti sul tema.

8) Sull'esempio di esperienze di successo in Europa e nei contesti locali come quelle gestite dalla Società della Salute della Valdera (Di Iacopo, 2012; Di Iacopo e Scarpellini, 2012) il contratto Agricoltura Sociale in rete consiste nella strutturazione di un partenariato locale aperto - realizzato tramite una piattaforma istituzionale - che condivida e promuova l'agricoltura sociale come strumento per lo sviluppo socio-economico solidale dell'area del parco, attraverso un sistema interdipendente e una governance a rete che metta in relazione: aziende agricole, servizi e istituzioni, terzo settore, consumatori, persone svantaggiate. Il progetto intende creare una struttura operativa nella quale il sistema di attori possa riconoscersi e nella quale possano incontrarsi la domanda e l'offerta di Agricoltura Sociale disponibili nell'area del Parco.

9) Il contratto il Carcere mette radici intende promuovere la trasformazione delle strutture penitenziare presenti nel territorio (Casa Circondariale Mario Gozzini, Casa Circondariale Sollicciano) da luoghi di pena e chiusura a luoghi di relazione e opportunità, sia per i soggetti sottoposti a misure cautelari e rieducative, i detenuti, sia per la comunità urbana esterna. I primi perché si possono giovare del lavoro terapeutico agricolo, i secondi perché possono usufruire della manodopera che i detenuti possono fornire presso le aziende agricole e per le esperienze che essi stessi possono svolgere nel carcere come cene, spettacoli, corsi misti per detenuti e studenti, orticoltura, partecipazione al lavoro negli orti comunitari. Inoltre, le mense degli istituti penitenziari potrebbero essere rifornite dalla rete del produttori locali dove gli stessi detenuti potrebbero lavorare.

10) Il contratto Passap-Orto promuove l'integrazione sociale tramite la nascita di aziende agricole, cooperative o orti condivisi interculturalimisti fra nativi e immigrati, profughi, rifugiati ecc. nei quali sviluppare la produzione di colture etniche per garantire il diritto alla cultura alimentare di ogni popolazione (Paddeu, 2012). I prodotti potrebbero rifornire anche i punti di vendita e di ristorazione etnica.

11) L'alloggio è un tema rilevante per coloro che intendono entrare nel mondo del lavoro agricolo senza possedere un'azienda di famiglia, soprattutto in aree in cui le prescrizioni urbanistiche da un lato prevedono la deruralizzazione e dall'altro non consentono di costruire nuovi edifici agrico1i. Perciò nell'area periurbana fiorentina è centrato il progetto Abitare solidale contadino, che prevede più linee di azione di cui la prima è quella di un housing sociale urbano-rurale che consenta ai giovani agricoltori di in- 
stallarsi nelle aree di margine urbano, riqualificandolo e consentendo al tempo stesso di coltivare le molte aree incolte nel territorio di pianura, in assonanza con alcune politiche francesi (Nougarès et al., 2014). Un'altra linea è quella di affiancare agli agricoltori anziani o comunque alle famiglie che non riescono a prendersi cura dei terreni agricoli (ma che ancora vivono in loco) soggetti svantaggiati disponibili a prendersi cura di tali terreni in cambio di ospitalità presso le case dei proprietari terrieri. Il progetto mira a trasformare il "problema abitativo", che oggi interessa fasce sempre più grandi di popolazione, in opportunità per alcuni soggetti e in generale a creare una comunità più coesa e che promuova un nuovo modello di assistenza agli anziani e cura degli spazi agricoli, mediante coabitazioni fondate sui principi del mutuo aiuto e della reciproca solidarietà.

12) L'ultimo contratto è quello relativo alla Villa La Guerrina Agricoltura comunitaria che per superare i pregiudizi verso il progetto comunitario in essere prevede la diffusione della conoscenza e il rafforzamento delle iniziative(sociali, ricreative, agricole) già presenti a Villa La Guerrina, una proprietà divenuta pubblica con un lascito ereditario che impone al Comune di Lastra a Signa l'utilizzo specifico per gli anziani. Il Comitato di autogestione delle terre pubbliche a Lastra a Signa infatti sta gestendo in forma comunitaria e continuativa le terre orticole e gli oliveti che circondano la Villa con l'accordo dell'Amministrazione comunale, mentre gruppi di anziani in forma saltuaria vengono accompagnati nell'edifico e nel giardino destinato. Il progetto intende integrare il progetto del Comitato con le altre attività degli anziani per promuovere la trasmissione intergenerazionale di competenze e saperi (nuove e antiche pratiche agricole) e la co-progettazione e co-gestione della filiera della produzione e trasformazione dei prodotti locali quali preparazione di marmellate, del pane, ecc., capaci di implementare reti di auto-aiuto, solidarietà e socialità nella comunità di Lastra. Villa La Guerrina come "passerella generazionale", economica e multiculturale, luogo di incontro, di conoscenza e di diffusioni di saperi che ruotano attorno all'agricoltura comunitaria.

\section{Conclusioni}

Le grandi contraddizioni a livello globale relative alla disparità di accesso e alla qualità del cibo (food security, food safety) trovano nei territori periurbani un contesto privilegiato di confronto e di innovazione. Le problematiche specifiche nelle città occidentali hanno caratteri marcatamente diversi rispetto alle aree del Sud del mondo e si caratterizzano per la messa in atto di progetti di "grande trasformazione" relativi alla riorganizzazione di un intero sistema insediativo in uscita dall'industrializzazione e dall' 
agroindustria il cui combinato disposto ha prodotto dei "deserti sociali" dove le relazioni interpersonali sono ridotte al minimo. Le aree periferiche sono aggregati monofunzionali privi di luoghi d'incontro e attraversati da grandi infrastrutture cieche che tagliano il tessuto minuto storico. Le nuove cattedrali sono i supermercati o i grandi centri commerciali che hanno inferto un duro colpo al commercio locale e alle reti corte del cibo. A partire da movimenti in un primo momento elitari, indirizzati alla ricerca di cibo biologico e locale, si è diffusa capillarmente nelle città occidentali una "rivoluzione del cibo" che non chiede semplicemente sicurezza o qualità, ma domanda sovranità alimentare, chiede di poter controllare e governare tutti i meccanismi della produzione, commercializzazione, distribuzione. Le Comunità del cibo sono ampie a variegate, raccolgono soggettività diverse, dai pensionati agli studenti, ai giovani intellettuali, ai disoccupati che sperimentano nuovi stili di vita e di consumo. Le aree periurbane sono diventale dei laboratori di innovazione di forme di riaggregazione sociale e di riqualificazione che vedono nella riterritorializzazione del cibo l'obiettivo condiviso che tiene assieme la ricerca della sostenibilità ambientale, della sicurezza alimentare e della giustizia sociale. I progetti integrati che perseguono un approccio bioregionale appaiono i più adeguati a questo scopo perché fondati sulla partecipazione sociale e sulla condivisione di uno spettro comune di valori orientati all'autogoverno delle comunità locali.

Durante la definizione del Progetto Coltivare con l'Arno. Parco Agricolo Perifluviale è emersa con nettezza la necessità di costruire un progetto in grado di rispondere al tempo stesso a più obiettivi come la riqualificazione ambientale, l'inclusione sociale per le fasce deboli, ma anche per chi ancora non è entrato a far parte del mondo dell'agricoltura, come i tanti giovani che non riescono ad accedere alla terra. In area periurbana la multifunzionalità è un'opportunità rilevante per costruire in forma innovativa una relazione stabile fra città e campagna come il ricorso all'abitare solidale contadino o la presenza di servizi rivolti espressamente alla città come il rifornimento delle mense pubbliche. Il processo mostra il vantaggio dell'essere impostato sul doppio livello di governance operativo (concertazione) e creativo (partecipazione) in grado co-progettare e di stringere accordi fra soggetti pubblici, privati e terzo settore come garanzia per l'attuazione futura e la co-govenance di quanto previsto nello scenario strategico.

\section{Riferimenti bibliografici}

Bastiani M., a cura di (2011). Contratti di fiume. Pianificazione strategica e partecipata dei bacini idrografici. Palermo: Flaccovio. 
Barmier H. and Morin X.K. (2012). Resilient urban community gardening programmes in the United States and municipal-third sector 'adaptive co-governance'. In: Viljoen A. and Wiskerke J.S.C. Sustainable Food Planning: Evolving Theory and Practice. Wageningen: Academic Publishers: 159-170, doi 10.3920/978-90-8686-187-3_13.

Bocchi S. (2015). Zolle. Storie di tuberi, graminacee e terre coltivate. Milano: Raffaello Cortina.

Butelli E. (2015). Tra Arno e colline: agricoltura qui vicino. Alimentazione sana, qualità della vita, rispetto dell'ambiente e del paesaggio. Un progetto di parco agricolo in riva sinistra d'Arno per Firenze, Scandicci e Lastra a Signa. Firenze: Sdt Edizioni.

Calthorpe P. and Fulton W. (2001). The regional city. Washington DC: Island Press.

Caritas Italiana (2012). I Ripartenti. Povertà croniche e inedite. Percorsi di risalita nella stagione della crisi. Rapporto 2012 sulla povertà e l'esclusione sociale in Italia. Roma: Caritas Italiana.

Di Iacovo F. (2012). La Costruzione delle politiche per l'agricoltura sociale in Europa. Impresa Sociale, 20(4): 124-147.

Di Iacovo F. e Scarpellini P. (2012). Dalla sperimentazione alla formalizzazione delle pratiche integrare. Il caso della Valdera in Toscana. Impresa Sociale, 20(4): 2002-210.

Donadieu P. (2006).Campagne urbane. Una nuova proposta di paesaggio della città. Roma: Donzelli.

Donadieu P. (2011). Agripolia, la città per i nostri figli. Eddyburg, <http://eddyburg.it/article/articleview/17618/0/307>.

Feagan (2007). The place of food: mapping out the 'local' in local food systems. Progress in Human Geography, 31(1): 23-42, doi: 10.1177/0309132507073527.

Frenkel S. (1994). Old theories in new places: environmentalism determinism and new regionalism. Professional Geographer, 46(3): 289-295.

Ginsborg P.A. (2010). Salviamo l'Italia.Torino: Einaudi.

Gottlieb R. and Joshi A. (2010). Food justice. Cambridge (MA): The MIT Press, <https://muse.jhu.edu/book/22133>.

Grillotti Di Giacomo M.G. (2012). Nutrire l'uomo, vestire il pianeta. Alimentazione-Agricoltura-Ambiente tra imperialismo cosmopolitismo. Milano: FrancoAngeli.

Iacoponi L. (2001). Sviluppo sostenibile e bioregione. QA - Rivista dell'Associazione Rossi-Doria, 4 <https://www.francoangeli.it $/$ riviste/Scheda_Rivista.aspx?IDArticolo $=17452 \&$ Tipo $=$ Articolo $\% 20$ PDF\&lingua $=i t \& i d R i v i s t a=25>$. 
Kloppenburg J., Hendrickson J. and Stevenson G.W. (1996). Coming in to the foodshed. Agriculture and Human Values, 13(3): 33-42.

McTaggart W.D. (1993). Bioregionalism and regional geography: place, people and networks. The Canadian Geographer, 37.

Magnaghi A., a cura di (2014a). La regola e il progetto. Un approccio bioregionalista alla pianificazione territoriale. Firenze: Firenze University Press, <http://www.fupress.com/archivio/pdf/2626_6705. pdf>.

Magnaghi A. (2014b). La biorégion urbaine. Petit traité sur le territoire bien commun. Paris: Eterotopia.

Magnaghi A. e Fanfani D. (2010). Patto città campagna. Un progetto di bioregione urbana per la Toscana centrale. Firenze: Alinea.

Meredith D. (2005). The bioregion as a communitarian micro-region (and its limitations). Ethics Place and Environment, 8(1): 83-94, doi: 10.1080/13668790500115755.

Mincke C. et Hubert M., éditeurs (2011).Ville et proximité. Bruxelles: Publications des Facultés Universitaires Saint-Louis.

Mougeot L.J.A., ed. (2005). Agropolis. The Social, Political, and Environmental Dimensions of Urban Agriculture. Earthscan and the International Development Research Centre.

Nougarès B., Candau J., Salles D. et Soulard C.-T. (2014). La gestion socio-spatiale du bâti agricole périurbain, un mode de régulation publique de la cohabitation entre activité agricole et usages résidentiels. Communication au Colloque SFER: Le foncier agricole: usages, tensions et régulations. Lyon, 11-12 Juin.

Oecd (2013). Rural-Urban Partnerships: An Integrated Approach to Economic Development. Bruxelles: Oecd Publishing, doi: 10.1787/ 9789264204812-en.

Paddeu F. (2012). L'agriculture urbaine dans les quartiers défavorisés de la métropole New-Yorkaise: la justice alimentaire à l'épreuve de la justice sociale. VertigO. La revue électronique en sciences de l'environnement, 12(2), <https://vertigo.revues.org/12686>.

Parham S. (2015). Food and Urbanism: The Convivial City and a Sustainable Future. London: Bloomsbury Publishing.

Poli D., a cura di (2013). Agricoltura paesaggistica. Visioni, metodi, esperienze. Firenze: Firenze University Press.

Poli D. (2014). Per una ridefinizione dello spazio pubblico nel territorio intermedio della bioregione urbana. In: Magnaghi A., a cura di. La regola e il progetto. Un approccio bioregionalista alla pianificazione territoriale. Firenze: Firenze University Press.

Poli D. (2015). Introduzione. Un parco agricolo multifunzionale come nuovo affaccio sul fiume per Firenze, Scandicci e Lastra a Signa. In: 
Butelli E., a cura di. Tra Arno e colline: agricoltura qui vicino. Alimentazione sana, qualità della vita, rispetto dell'ambiente e del paesaggio. Un progetto di parco agricolo in riva sinistra d'Arno per Firenze, Scandicci e Lastra a Signa: 5-11. Firenze: Sdt Edizioni.

Poli D. (icp). Campagne insorgenti. Agricoltura contadina e 'bene comunitario' nella fattoria di Mondeggi a Firenze. Archivio di Studi Urbani e Regionali.

Pollan M. (2006). The Omnivore's Dilemma: A Natural History of Four Meals. New York: Penguin Press. Trad. it. (2008). Il dilemma dell'onnivoro. Milano: Adelphi.

Pothukuchi K. and Kaufman J.L. (1999). Placing the food system on the urban agenda: the role of municipal institutions in food systems planning. Agriculture and Human Values, 16(2):213-224, doi: 10.1023/A: 1007558805953.

Potito M. e Borghesi R. (2015). Genuino Clandestino. Viaggio tra le agriculture resistenti ai tempi delle grandi opera. Firenze: Terra Nuova.

Soja E.W. (2009). The city and spatial justice/La ville et la justice spatiale". Justice spatiale/Spatial justice, $1<$ http://www.jssj.org>.

Sonnino A. (2014). I limiti della Risorsa terra e delle altre risorsa naturali. EAI Energia, Ambiente e Innovazione, 6: 8-19, doi: 10.12910/EAI2 014-102.

Stierand Ph. (2012). Food Policy Council: recovering the local level in food policy. In: Viljoen A. and Wiskerke J.S.C. Sustainable Food Planning: Evolving Theory and Practice. Wageningen: Academic Publishers: 67-78, doi 10.3920/978-90-8686-187-3_13.

Thayer R.L. (2003). Life Place: Bioregional Thought and Practice. Oakland (CA): University of California Press. 